\title{
Sources of student engagement in Introductory Physics for Life Sciences
}

\author{
Benjamin D. Geller, ${ }^{1}$ Chandra Turpen, ${ }^{2}$ and Catherine H. Crouch ${ }^{1}$ \\ ${ }^{1}$ Department of Physics and Astronomy, Swarthmore College, Swarthmore, Pennsylvania 19081, USA \\ ${ }^{2}$ Department of Physics, University of Maryland, College Park, Maryland 20742, USA
}

(Received 19 May 2017; published 4 April 2018)

\begin{abstract}
We explore the sources of student engagement with curricular content in an Introductory Physics for Life Science (IPLS) course at Swarthmore College. Do IPLS students find some life-science contexts more interesting than others, and, if so, what are the sources of these differences? We draw on three sources of student data to answer this question: (1) quantitative survey data illustrating how interested students were in particular contexts from the curriculum, (2) qualitative survey data in which students describe the source of their interest in these particular contexts, and (3) interview data in which students reflect on the contexts that were and were not of interest to them. We find that examples that make interdisciplinary connections with students' other coursework in biology and chemistry, and examples that make connections to what students perceive to be the "real world," are particularly effective at fostering interest. More generally, students describe being deeply engaged with contexts that foster a sense of coherence or have personal meaning to them. We identify various "engagement pathways" by which different life-science students engage with IPLS content, and suggest that a curriculum needs to be flexible enough to facilitate these different pathways.
\end{abstract}

DOI: 10.1103/PhysRevPhysEducRes.14.010118

\section{INTRODUCTION}

\section{A. Motivations for Introductory Physics for Life Science (IPLS)}

Although attempts have been made for decades to reform introductory physics to better meet the needs of a growing population of biology and pre-health-profession students $[1,2]$, there has been a renewed focus on these efforts in recent years [3-6]. These efforts have come in response to a series of national calls to better train future physicians, biologists, and medical researchers in physics, calls that emphasize the increasingly prominent position that physical tools and technologies occupy in the life-science toolkit [7-9]. In particular, the motivation for these reform attempts has largely been framed in terms of the importance of training life-science students in the quantitative reasoning and physical and computational modeling skills that are required of them as they move on to upper-division coursework and modern careers in clinical research and medicine.

At the same time, physics instructors who teach lifescience students are motivated not just by this desire to better equip life-science students for their future

Published by the American Physical Society under the terms of the Creative Commons Attribution 4.0 International license. Further distribution of this work must maintain attribution to the author(s) and the published article's title, journal citation, and DOI. careers-what we might call "workforce motivations"but also by a desire for their courses to be interesting and engaging to the life-science student population. In practice, instructors recognize the importance of positive affective experiences as both a mediator of participation in and an outcome of Introductory Physics for Life Science (IPLS) courses. Because life-science students often have negative orientations towards physics [10,11], helping such students come to appreciate or like physics is seen as an important component of interdisciplinary learning $[12,13]$. This goal is sometimes made explicit, but more often is conveyed implicitly through efforts to include content that biologists or health professionals would find "exciting" or of particular "interest." However, the sources of these affective responses have not been well researched.

\section{B. Interest and engagement}

Our analysis of student experiences in the IPLS environment relies on student responses to survey and interview prompts that include the words interest and engagement. On surveys, we asked students to assess the degree to which each of the life-science contexts ${ }^{1}$ in the course "sparked their interest," and in interviews we asked students to speak about contexts that were "particularly engaging." We therefore rely

\footnotetext{
${ }^{1}$ Throughout this paper, life-science "contexts" refers not to any single problem or example, but rather to the most important biological applications around which each physical unit was constructed (see Sec. II).
} 
to a considerable extent on the students' own interpretations of these words, and we recognize that different students might interpret the words interest and engagement differently. Nevertheless, we as researchers need to be clear about the meanings that we associate with these words, and about the relationship between them. We can then determine whether the responses we get from students are at least consistent with these working definitions.

In the educational psychology literature, interest has a dual meaning: (a) the psychological state of a person participating in some activity (say, the psychological state of a student working on an IPLS physics problem) and (b) the motivational predisposition of the person to reengage with that activity over time [14]. For the purposes of this study, we focus primarily on the former meaning, the psychological state of the student at a particular moment in time. At any given moment, the psychological state of a student who is "interested" is characterized by "increased attention, effort, concentration, and affect during engagement" [14]. Since we do not report on real-time observations of students as they work through course materials, we rely on students' retrospective assessments of their own psychological states when they were engaging with the materials.

Engagement refers to a student's "involvement in a particular context" (say, involvement in trying to solve a problem on an IPLS homework assignment) [14]. If a topic or activity is interesting, it is almost certainly engaging. However, engagement need not indicate that a student is interested. One can imagine many reasons why one might be engaged in a physics problem, such as an external motivation to get a good grade, that are not tied to a particular interest in the problem. For the purposes of this study, we focus on engagement that is directly tied to interest. More specifically, we focus on engagement that stems from interest in particular biological contexts. Student responses in which positive affect is tied directly to the specifics of particular curricular contexts are taken as evidence that such engagement exists.

We view neither interest nor engagement as stable states that a student carries with them from moment to moment. Instead, both of these dimensions are influenced by the different contexts in which an individual participates [15]. This framework does not preclude the possibility that these states may be more or less consistently activated across a variety of contexts. It simply starts from the assumption that these states are sensitive to context and leaves the determination of whether they are more or less stable across context to future empirical investigation. When we make claims about interest in or engagement with curricular content, those claims are valid for particular students at particular moments of time and in particular curricular contexts.

Affective dimensions of student learning are worthy of our attention for two reasons. First, the educational psychology literature has demonstrated that interest is an

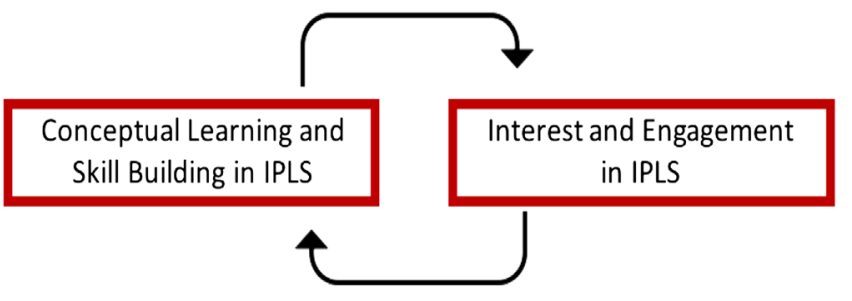

FIG. 1. Interest and engagement can enhance students' attention, effort, and conceptual learning (bottom arrow). At the same time, positive affective experiences stemming from particular aspects of the IPLS curriculum can be ends in and of themselves (top arrow).

important factor in enhancing students' attention, effort, and conceptual learning [16-18]. In other words, interest and engagement are mechanisms by which to improve the development of professional skills that are at the heart of the workforce motivations for IPLS. At the same time, we value the affective experience as an important student learning outcome in and of itself [12]. Just as interest and engagement support conceptual learning and the development of skills, the manner in which conceptual learning and skill building take place influences how and whether students become interested and engaged (Fig. 1).

The focus in this paper is on the top arrow in Fig. 1, on the way in which curricular and pedagogical choices contribute to greater student interest and engagement in the IPLS curriculum.

\section{Prior work on interest in the context of Swarthmore IPLS}

Prior work has shown that life-science students at Swarthmore provide more expertlike responses to the Colorado Attitudes about Science Survey (CLASS) at the end of a second-semester IPLS course than they do at the end of a first-semester traditional (not IPLS) physics course [19-21]. More specifically, whereas life-science students' attitudes declined during the standard firstsemester course, as measured by CLASS scores, those students' attitudes toward physics hold steady or are improved by the IPLS course. Furthermore, items from the CLASS that map onto indicators of student interest in physics have been examined specifically [22], and it was found that students who came into the IPLS course with low levels of interest in physics show significant improvements over the course of the IPLS semester [20,21]. In this paper we explore more concretely the sources of this greater interest. Our goal is to determine whether particular IPLS curricular contexts resulted in higher interest scores, and to explore the underlying factors that contribute to these differences. Specifically, this paper seeks to expand on prior work by answering two research questions:

(1) Do IPLS students find some life-science contexts more interesting than others? 
(2) What are the sources of these differences; i.e., what makes some life-science contexts more interesting than others?

In the following section we describe the IPLS pedagogical structure at Swarthmore, a structure that we believe serves as a foundation for fostering student interest. That is, we identify the pedagogical features and modes of student interaction that we believe are essential for obtaining the overall levels of engagement with the curriculum that we observe. Having described a particular pedagogical setting that fosters student engagement, Sec. IV then discusses how and why, within this setting, some biological contexts resonated more strongly with students than others.

\section{SETTING FOR THE STUDY: IPLS AT SWARTHMORE}

\section{A. Student population}

All of the students in our study were life-science, chemistry, or pre-health-profession students enrolled in a second-semester IPLS course on electricity, magnetism, and optics. Most of these students had previously taken a traditional first-semester mechanics course without biological emphasis. ${ }^{2}$ Unless otherwise indicated in the rest of the paper, the terms "IPLS course" or "IPLS" refers to the second-semester electricity, magnetism, and optics course in which all students in the study were enrolled. The IPLS course was nominally calculus based, and had a semester of calculus as a prerequisite, but in practice very little calculus was used and students were rarely asked to evaluate integrals. Although there were no formal biology or chemistry prerequisites for the course, the vast majority of students in the course had taken or were coenrolled in both biology and chemistry coursework. In particular, it was quite common for students to have taken at least one semester each of introductory biology and general chemistry, two semesters of organic chemistry, and one intermediate-level biology course prior to enrolling in IPLS.

Enrollment in the IPLS course averaged about 50 students over the five years of the study, each class consisting of approximately $10 \%$ freshmen, $75 \%$ sophomores and juniors, and 15\% seniors. One instructor (coauthor Crouch) taught the first three iterations of the course included in this study (years 1-3), and a different instructor (coauthor Geller) taught the last two iterations (years 4 and 5).

\footnotetext{
${ }^{2}$ In the Fall 2015 semester Swarthmore did offer an IPLS version of the first-semester mechanics course, so students interviewed during the last year of the study had taken this new course prior to enrollment in the second-semester IPLS course. All of the survey data, however, are from students who had taken a traditional (non-IPLS) first-semester mechanics course.
}

\section{B. Pedagogical structure}

We view particular pedagogical features of the IPLS course and particular ways in which students were asked to participate in the course as essential for achieving the overall levels of engagement that we observed. We would not expect to see high engagement without these pedagogical features, and without such a foundation of engagement it would not have been possible to explore how and why some biological contexts resonated more strongly with students than others.

Cognitive apprenticeship forms the pedagogical framework for the IPLS course at Swarthmore [23]. The goal of traditional apprenticeship is for the learner to develop skills in the same environment in which an expert uses them. Such an environment should constantly prompt the apprentice to assess (i) why they are learning what they are learning and (ii) how what they are learning connects to things they already know. Within the cognitive apprenticeship framework, the classroom is meant to simulate this sort of environment as closely and as frequently as possible. In the context of the IPLS course at Swarthmore, such an environment was achieved by incorporating rich and authentic biological contexts into the core of the course. The course foregrounded the modeling of authentic biological systems and emphasized that the use of simplified physical models was important for making quantitative and qualitative predictions about complex biological systems.

The cognitive apprenticeship framework involved establishing an environment of expert practice and iterating among the instructional approaches of modeling, coaching, and fading [24]. These approaches were all supported by varying degrees of scaffolding. The IPLS course was lecture based, but both instructors had experience with student-centered pedagogies and included elements of interactive peer instruction in almost every class session [25]. The instructors routinely demonstrated the process of complex problem solving as part of the interactive lecture, with particular attention given to the decision-making steps and simplifying assumptions that are essential for describing complex biological systems with simple physical models. Explicit articulation of these decision-making steps was an essential aspect of the modeling stage of the apprenticeship.

Clicker-question ConcepTests were used throughout most lectures and discussion between students was encouraged and facilitated by undergraduate peer assistants [25]. This interactive lecture environment facilitated the coaching stage of apprenticeship, as peers and formal peer educators coached students during both the discussion of conceptual questions in lecture and during structured group problem-solving sessions outside of class. All students were also enrolled in weekly three-hour lab sections, about a third of which were "workshop recitations" in which students completed extended tutorial-like activities and made experimental observations in small 
groups. Activities and problems included scaffolding that helped students succeed when they were still learning a skill. This scaffolding was not only content specific, but was also meant to remind students of the general problemsolving strategies to which they were introduced in lecture. Finally, this scaffolding gradually faded away as students progressed through a unit and through the course as a whole, allowing them to develop greater independence and proficiency. All of these steps were iterative, as the more proficient students became, the more they gained from the modeling and coaching portions of the apprenticeship [24].

\section{Curricular design}

To create an environment of expert practice, each topic in the course was motivated by and organized around a central biological context. Curricular design was broadly governed by the principles of authenticity [26] and expansive framing $[27,28]$. Authenticity refers here to the curricular goal that students would perceive the curricular contexts to be meaningful ones, rather than attempts to find biological meaning where none actually existed (we do not, for example, consider the replacement of a car with an animal in a standard kinematics problem to be authentic). Expansive framing refers to the goal of presenting the conceptual material in a way that would be seen by students to be broadly applicable to the scientific community outside of the physics classroom, and to students' future interests and careers. In exploring these contexts, the instructor encouraged students to draw on their own backgrounds in biology and chemistry, and explicitly positioned the students as having expertise in areas with which they as physics instructors might have little familiarity [29].

The content and organization of the course is described in detail elsewhere [25]. The key curricular feature of the course is the organization of each physical unit around one or two key biological contexts. These contexts were not designed as optional "add-on" applications to be tackled only after the core physical ideas were learned in a traditional way; rather, they were integral to the course and repeatedly referred to throughout each unit as the physical ideas were developed. Table I shows the key examples investigated in each unit of the course.

Students were expected to iteratively engage with these biological contexts in multiple ways: through conceptual questions posed during the interactive lecture, through problems on homework sets that went beyond the basic ideas presented in lecture, and through context-rich scenarios posed during recitation and lab sessions. Students were assigned problems that foregrounded a biological driving question (e.g., why does an action potential need to be regenerated along a neural axon?), and the core physics (e.g., resistance and capacitance in simple electrical circuits) was presented as an essential tool for arriving at both qualitative and quantitative answers to the driving question. Models were often developed in an iterative fashion as the
TABLE I. Each physical unit in the Swarthmore IPLS course was organized around a small number of key biological contexts.

\begin{tabular}{ll}
\hline \hline Physics content & \multicolumn{1}{c}{ Biological contexts } \\
\hline Electrostatics & Cell membrane potential \\
Circuits & $\begin{array}{l}\text { Charge flow across the cell membrane } \\
\text { Nerve signaling } \\
\text { Magnetic sensing } \\
\text { Magnetism }\end{array}$ \\
& $\begin{array}{l}\text { Muclear magnetic resonance } \\
\text { Animal vision and vision correction }\end{array}$ \\
Geometric optics & $\begin{array}{l}\text { Microscopy } \\
\text { Echolocation }\end{array}$ \\
\hline \hline
\end{tabular}

course progressed. For example, the model of a cell membrane was developed gradually as new electrical ideas (resistance, capacitance, current) were encountered. In this way, each time a new physical property was described, students immediately encountered its relevance for modeling a real biological system.

The topics in Table I were chosen by coauthor Crouch after consulting with a select number of biology and chemistry faculty at Swarthmore. These faculty members provided insight into some of the topics and skills that they would like to see their students develop in the IPLS course, and advised on which traditional second-semester introductory physics topics could be deemphasized or eliminated in order to create space for the new biological material. This close coordination of the IPLS instructor with other faculty in the natural science division was important for developing a course that could foster coherence across the curricula.

Coauthor Crouch taught the geometrical and physical optics units first, before moving on to electricity and magnetism. Coauthor Geller taught electricity and magnetism before moving on to the geometrical and physical optics units. Coauthor Geller also included several lectures at the end of the IPLS course that focused on modern physics (special relativity and quantum mechanics) in a conceptual way. In addition to these sequencing differences, the two instructors differed in the frequency with which they delivered explicit messaging about interdisciplinary learning. ${ }^{3}$

\section{METHODOLOGY}

We draw on three data sources in exploring the curricular sources of student engagement in our IPLS course: (1) quantitative survey data in which students report their level of interest in particular contexts from the curriculum,

\footnotetext{
${ }^{3}$ This claim about differences in explicit messaging comes largely from observations made by each instructor of the other's course.
} 
(2) qualitative survey data in which students describe the source(s) of their interest in these particular contexts, and (3) interview data in which students reflect on the topics that were and were not of interest to them.

\section{A. Survey data}

We asked students on an end-of-semester survey to rank their level of interest in the most prominent life-science contexts discussed in the course. Specifically, we asked students to assess the degree to which each of the lifescience contexts sparked their interest, using a five-point Likert scale from 1 (the life-science contexts "did not spark my interest at all") to 5 (the life-science contexts "greatly sparked my interest"). ${ }^{4}$ We combined and analyzed Likertscale responses from four iterations of the course (years $1-4, N=194$ responses). We do not include data from year 5 of the study because, as noted earlier, many year 5 students had taken a new IPLS version of first-semester mechanics prior to enrollment in the second-semester IPLS course. To ensure a valid comparison across years, all survey data are from students who had taken a traditional (non-IPLS) first-semester mechanics course.

On the same end-of-semester survey, following the question asking students to provide Likert-scale assessments of their interest level in various life-science contexts, we provided an open-ended prompt asking students to "say a little bit about how the use of the life-science examples did or did not spark your interest." responses from four iterations of the course (years 1-4, $N=175$ responses), and categorized responses into one of three groups: "rich and nuanced positive responses," "vague positive responses," and "negative or neutral responses" (see Table II). The unit of analysis was an

\footnotetext{
${ }^{4}$ In addition, we asked the students to assess the degree to which the life-science contexts "sustained" their interest, using a fivepoint Likert scale from 1 (the life-science contexts "did not sustain my interest at all") to 5 (the life-science contexts "greatly sustained my interest"). The phrasing for these questions was motivated by the work of Renninger and Hidi on the four stages of interest development [12], and we theorized that it was possible that students would see some contexts as helpful in sparking their interest and others as more helpful in sustaining it. Perhaps not surprisingly, given the subtle difference in wording of the two questions and the unlikelihood that students would even distinguish the meanings of "spark" and "sustain" in a useful way, we found no meaningful differences between the students' responses to the two forms of the question. In terms of both the numerical scores on the Likert scale and their subsequent qualitative descriptions (see below), students did not seem to distinguish between the two question phrasings, and very similar data were acquired from both versions. As such, here we analyze only data obtained from the "sparked" version of the question.

${ }^{5}$ Although we included both a "sparked" and "sustained" version of this open-ended prompt, just as we did for the Likert-scale questions, we again saw no meaningful differences in how students responded to the two versions and therefore do not include the "sustained interest" responses in our analysis.
}

TABLE II. Responses to an open-ended prompt asking students to "say a little bit about how the use of the life-science examples did or did not spark your interest" were coded and categorized into one of three groups. Qualitative responses from four iterations of the IPLS course (years $1-4, N=175$ responses) were analyzed, and the percentage of responses belonging to each group is shown.

\begin{tabular}{lc}
\hline \hline Type of response & Percentage of responses \\
\hline Rich or nuanced positive & 60 \\
Vague positive & 25 \\
Negative or neutral & 15 \\
\hline \hline
\end{tabular}

individual student's response, as responses were not long enough to be broken into multiple segments.

As shown in Table II, a large majority of student responses (about 85\%) indicated that the inclusion of life science contexts in the courses contributed positively to overall interest in the course. At the same time, about $15 \%$ of the responses were categorized as "negative or neutral." That is, about $15 \%$ of the life-science student responses to the open-ended survey prompt described the life-science contexts as either irrelevant to their engagement in the course or as a negative feature of the course. Frequently these student responses indicated that the applications to medicine or biology were distracting, making it harder for students to focus on the key physical principles. All the remaining $85 \%$ of student responses were positive about the role that the life-science contexts played in the course, but about $25 \%$ of the total responses analyzed were too vague as to be analyzed further according to a coding scheme. For example, some students responded that the life-science examples sparked their interest or made the course more engaging because "they were more relevant," but did not elaborate in any meaningful way on why the examples were relevant. In other words, student statements were categorized as "vague positive responses" when they just restated the question in different terms but did not unpack the source of their interest. Having separated out the $15 \%$ of responses that were negative, and the $25 \%$ of responses that were too vague to be analyzed in greater detail, the remaining 60\% of the responses (the "rich and nuanced positive responses") were coded further.

We identified five sources of interest that appeared repeatedly in the student responses to the open-ended prompt. While these five sources are not mutually exclusive (indeed, a small number of responses were found to contain more than one source and were accordingly coded into more than one category), they did cover the entire space of nuanced student responses that we received. We designed a coding scheme based on these five sources, and two authors (Geller and Crouch) independently coded all the "rich and nuanced positive responses" according to the scheme. The two authors assigned the same codes to the vast majority of the student responses (Cohen's $\kappa=0.71$ ). Because the 
coding disagreements between the two authors were so minimal, and could be easily resolved upon discussion of the few individual cases where different codes were used, no adjustment of the original coding scheme was necessary. Instead, in the very small number of cases where there was disagreement, both authors' code assignments were included when tallying frequencies.

\section{B. Interview data}

To gain a more nuanced understanding of the sources of student engagement with the IPLS curriculum, we conducted a series of student interviews over the final two years of the study (2014-2016). In total, 30 hour-long interviews were conducted with 14 different students over the two academic years. An outside physics education research (PER) expert (Turpen) with considerable experience in conducting undergraduate student interviews came to campus to conduct interviews at the beginning and end of the IPLS semester during each of the two academic years. ${ }^{6}$ All students in the IPLS course were invited to participate in the interviews. Among those who responded to the open invitation, students were selected by the outside PER expert in order to achieve approximate gender balance and in order to interview students with a variety of academic majors. During the first of the two years, the seven students interviewed included three biology majors, a neuroscience major, a chemistry and mathematics double major, and two students majoring in the social sciences who were taking the course to fulfill the pre-med requirements. During the second year, the seven students interviewed included three biology majors, one biochemistry major, one neuroscience major, and two chemistry majors. The average final exam score for the 14 students interviewed during the final two years of the study (81\%) was comparable to the average final exam score for all 106 students enrolled in the course during those two years $(78 \%)$.

The interviews were semistructured and focused on the nature and source of student engagement in the traditional and IPLS environments. Although the research team analyzed all of the interviews, most of the data in this paper come from the interviews conducted at the end of the second-semester IPLS course, after students had taken a full year of introductory physics. In those end-of-semester interviews, students were given open-ended prompts such as "what biological contexts from the course stood out to you as particularly engaging?" and "what made those contexts engaging?" Follow-up questions asked students to elaborate further on the source of their engagement, or to provide more specific examples.

\footnotetext{
${ }^{6}$ In addition, during the second academic year when a firstsemester IPLS course was offered for the first time, the interviewer came to campus to conduct interviews in the middle of that first-semester course as well.
}

All interviews were transcribed and analyzed by a research team consisting of this paper's authors and several undergraduate summer researchers. Themes were identified across interviews with different students, as well as longitudinally within the set of interviews associated with individual students. These themes emerged empirically as a means for understanding and interpreting our data, rather than as preexisting theoretical constructs. The goal of the interview analysis was not to count the number of individual student statements belonging to a particular theme (because the number of interviewed students was small, this would not be a particularly meaningful metric). For this reason, interviews were not "coded" in the way that the open-ended survey responses were coded. Rather, the goal of the interview analysis was to identify broad themes that emerged across the set of interviews as a whole, themes which could help us further unpack the source of student interest as measured in the survey responses. We came to refer to these broad themes as "engagement pathways." The analysis of interview data was iterative; the definitions of the engagement pathways became more refined over time, as the research team progressively identified the minimal set of pathways that fully captured the most salient aspects of the interviews.

This paper reports on the common themes that emerged across interviews conducted with different students, and is not focused explicitly on the evolution of this engagement for individual students during the course of the year.

\section{RESULTS}

\section{A. Quantitative survey results}

Students reported having greater interest in some lifescience contexts in the course than others (Fig. 2). While students reported having at least moderate interest in all of the contexts, with no topic receiving an average score of less than " 3 " on the five-point Likert scale, there were significant differences between contexts. These relative rankings were consistent across multiple offerings of the course by a single instructor, and across versions of the

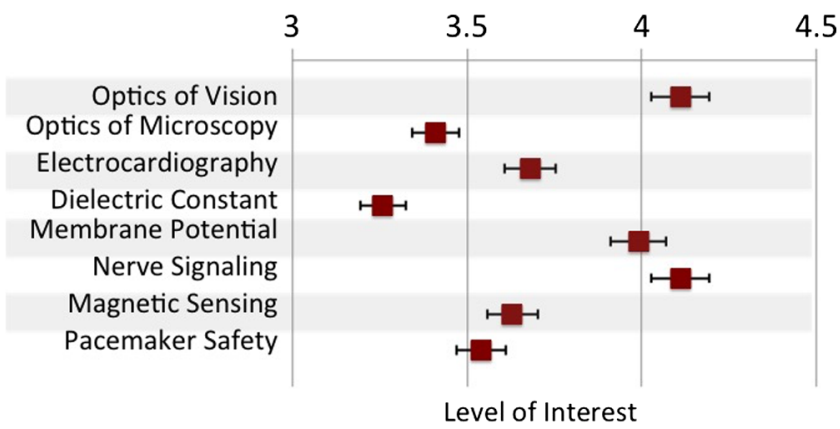

FIG. 2. Students ranked their levels of interest in key biological contexts on a five-point Likert scale. Average results and standard errors are shown for the IPLS course (years $1-4, N=194$ responses). 
TABLE III. Students ranked their interest in the "dielectric constant" topic differently, depending on whether or not they had encountered the topic in their biochemistry course. Average fivepoint Likert-scale ratings and standard errors are shown for the IPLS course (Years $1-4, N=194$ responses).

\begin{tabular}{lc}
\hline Students' prior experience & Average interest rating \\
\hline Students who had taken biochemistry & $3.6 \pm 0.1$ \\
Students who had not taken biochemistry & $3.1 \pm 0.1$ \\
\hline \hline
\end{tabular}

course offered by two different instructors, despite the differences in sequencing and interdisciplinary messaging between the two instructors that was mentioned in Sec. II.

The results in Fig. 2 were initially surprising. Consider, for example, the high scores routinely given to the "membrane potential" and "nerve signaling" topics. We did not necessarily predict that a class composed mostly of pre-health-profession students would find these contexts to be of greater interest than topics like "electrocardiography" or "pacemaker safety." The relative scores reported for "the optics of vision" and "optics of microscopy" examples are also intriguing. While both of these topics involve the same physical principles (geometric optics), and occupied the same number of lectures in the course, students consistently ranked the optics of vision as more interesting than they did the optics of microscopy.

Another noteworthy result is the particularly low score given to the topic of the "dielectric constant." Outside of the IPLS course, the dielectric constant is a concept discussed only in an intermediate-level biochemistry course that some but not all of the students in the course had taken or were taking concurrently with IPLS. For this reason, we divided student interest rankings for the "dielectric constant" topic between those who had and had not been exposed to a treatment of the dielectric constant in biochemistry. We found that students who had encountered the dielectric constant in biochemistry ranked the IPLS treatment of the topic more highly than their classmates who were seeing it for the first time (Table III).

We will return to the results in Fig. 2 once we have examined the reasons that students give for finding some contexts more interesting than others. That qualitative data will help us to understand the relative rankings that students gave for the various examples.

\section{B. Qualitative survey results}

In reading the student responses to the open-ended prompt about what made the life-science contexts interesting, we identified five categories that appeared repeatedly. While these categories are not mutually exclusive (indeed, some responses were found to belong in more than one category and were coded as such), they did cover the entire space of "rich and nuanced positive responses" that we received. That is, all of the nuanced responses fell into at least one of these categories. The five categories are defined as follows ${ }^{7}$ :

Real-world application.-Students described being interested in the examples because they saw them as relevant for better understanding the "real world," where this real world included their own academic experiences in other science classrooms.

Ease and accessibility.-Students described being interested in the examples because they made the course seem easier and/or more conceptually accessible.

Future career relevance.-Students described being interested in the examples specifically because they would be of relevance to their future careers or livelihoods.

Underlying mechanism.-Students described being interested in the examples because they illustrated "how things work," often in the context of biomedical instrumentation or technology.

Interdisciplinary connections. - Students described being interested in the examples because they connected to contexts encountered in biology and chemistry coursework.

To illustrate these categories concretely, Table IV includes example student responses that were coded as belonging in each category, as well as key words or phrases that often appeared in responses belonging to each. It was not a requirement that these key words or phrases be present in order for a response to be coded in a particular category, but because they did appear frequently they are listed to better articulate the categories.

The frequency with which student responses were coded as belonging to each of the five categories is shown in Fig. 3.

As shown in Fig. 3, students were least likely to attribute their interest in the life-science contexts to career relevance or to the sense that these contexts made the course easier. This is not to say that factors like future career relevance are unimportant to this population of students. One could imagine, for example, that future career relevance is why many students were enrolled in the course in the first place, so in a broader sense it is indeed an important source of engagement. But here, in the context of an anonymous IPLS end-of-semester survey where they were asked to reflect on the source of their interest, students did not mention their future careers particularly often.

Students did, however, frequently attribute their interest in life-science contexts to connections made with their other coursework. They were excited by examples that touched on contexts encountered in their other coursework, and frequently referenced "synergy" with other courses as a driver of their interest in IPLS. They seemed to especially appreciate life-science contexts that facilitated interdisciplinary connections with their biology and chemistry

\footnotetext{
${ }^{7}$ Raw student responses to this survey prompt are included in the Supplemental Material [30].
} 
TABLE IV. Five categories appeared repeatedly in student responses to the open-ended prompt about the source of their interest in the life-science contexts. Example student statements and keywords are provided to better articulate the category meanings.

\begin{tabular}{|c|c|c|}
\hline Category & Examples & Key words or phrases \\
\hline $\begin{array}{l}\text { Real-world } \\
\text { application }\end{array}$ & $\begin{array}{l}\text { The [life-science] examples gave me a better understanding of how physics could be } \\
\text { applied to things in the real world, since otherwise a lot of it seems really } \\
\text { theoretical... } \\
\text { Using life-science examples to explain physics makes physics seem a little bit more } \\
\text { tangible and applicable... }\end{array}$ & $\begin{array}{l}\text { "relevant" } \\
\text { "real world" } \\
\text { "applied" }\end{array}$ \\
\hline $\begin{array}{l}\text { Ease and } \\
\text { accessibility }\end{array}$ & $\begin{array}{l}\text { Using life-science examples makes the concepts easier to understand... } \\
\text { The life-science examples made the physics content more accessible... }\end{array}$ & $\begin{array}{l}\text { "easy" } \\
\text { "understandable" }\end{array}$ \\
\hline $\begin{array}{l}\text { Future career } \\
\text { relevance }\end{array}$ & $\begin{array}{l}\text { As a prospective future physician, I mainly took physics because it was required... the } \\
\text { life-science applications, however, always piqued my interest because I thought } \\
\text { that some day this information might be useful to me-beyond the MCAT... } \\
\text { I enjoyed learning about biomedical applications because I believe that they will be } \\
\text { useful in the future when I (hopefully) pursue a career as a physician... }\end{array}$ & $\begin{array}{l}\text { "need to know for my } \\
\text { career" "useful in the } \\
\text { future as a doctor" }\end{array}$ \\
\hline $\begin{array}{l}\text { Underlying } \\
\text { mechanism }\end{array}$ & $\begin{array}{l}\text { I wanted to understand how glasses work, as well as how MRI machines work, which I } \\
\text { was able to do with the help of these examples... } \\
\text { In my chemistry classes, we don't really talk about how certain instruments work, just } \\
\text { that they do. I found it very useful to talk about the NMR and what is actually going } \\
\text { on inside of it... }\end{array}$ & $\begin{array}{l}\text { "how it works" "what is } \\
\text { actually going on" }\end{array}$ \\
\hline $\begin{array}{l}\text { Interdisciplinary } \\
\text { connections }\end{array}$ & $\begin{array}{l}\text { The life-science examples did a great job tying together my understanding of } \\
\text { biochemistry, microbiology, and physics... } \\
\text { The examples showed me that there is great synergy between the physics and biology } \\
\text { explanations... } \\
\text { The examples helped me to apply physics to topics we were studying in other classes. } \\
\text { For example in both biochemistry and physics, we looked at salting out of DNA, } \\
\text { and in a different way for each class. }\end{array}$ & $\begin{array}{l}\text { "connected" "tied together" } \\
\text { "synergy" }\end{array}$ \\
\hline
\end{tabular}

classes, a sentiment that will be explored in greater detail below when describing the results of the interviews.

Students also saw the life-science examples as connecting to the real world, as evidenced by the large number of responses that were coded as "real-world application." However, we see in these qualitative responses that many life-science students include their nonphysics academic experiences as examples of "real-world" experiences when considered in the context of a physics course. As defined

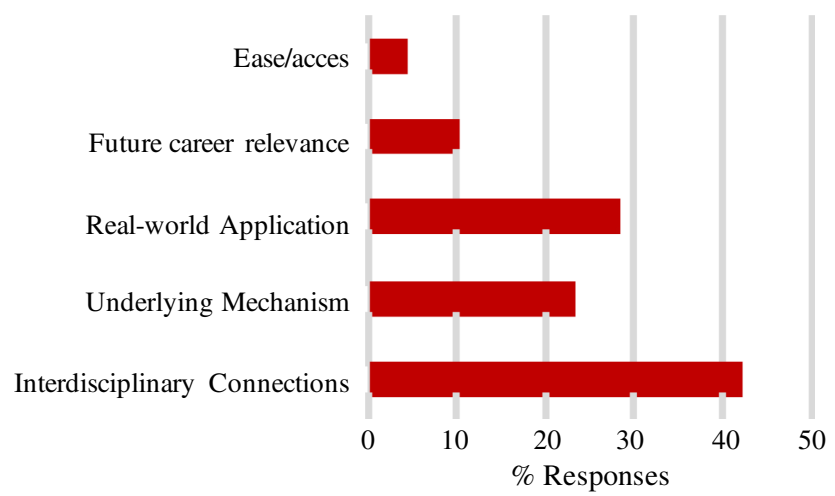

FIG. 3. The percentages of "rich and nuanced positive student responses" coded into each of the five categories of Table III are displayed. Data are shown for the IPLS course (years $1-4, N=$ 175 responses). here, the "real world" should therefore not be understood only as "everyday experience," but also as including aspects of students' academic lives outside of IPLS. For example, students regularly used "real world" in their openended survey responses when referring to phenomena encountered in biology class. It is worth emphasizing this last point, since it is sometimes implicit in the analysis of attitudinal surveys like the CLASS that all students would mean roughly the same thing by "everyday experience." We will return to discuss Fig. 3 in more detail after describing the data obtained from a series of student interviews.

\section{Interview results}

We see from the qualitative survey data (Fig. 3) that students reported a high level of interest in IPLS curricular materials that connected to their other science coursework ("interdisciplinary connections") or had relevance to what they perceive to be the real world ("real-world applications"). The interview data allow us to go one step further, to begin to unpack why students cite such categories as particularly important.

Specifically, the interview data allow us to locate some of the categories from Fig. 3 within two more general sources of engagement with the course-coherence and personal meaning (Fig. 4). As shown in Fig. 4 and described below, we identify "interdisciplinary connections" as one way in 


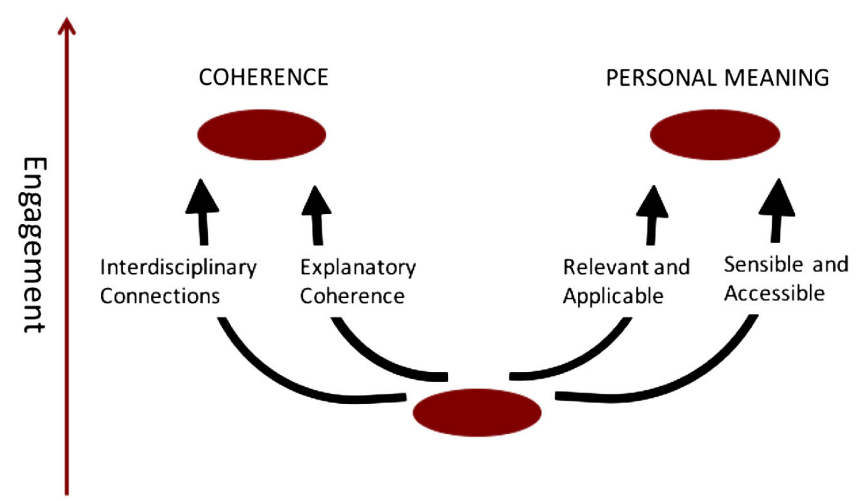

FIG. 4. The students we interviewed described becoming more highly engaged with IPLS curricular content for a variety of reasons. Students described high levels of interest in contexts that fostered a sense of coherence (left-hand side) or a sense of personal meaning (right-hand side). The most prominent "engagement pathways" (black arrows) are shown, and labeled by the factor that is key in facilitating increased engagement relative to the student's initial, pre-IPLS state. The pre-IPLS state is represented by the middle red oval from which all the pathways emerge, and the meaning of each factor is unpacked below.

which students achieve a sense of coherence in the course, and "real-world applications" as one way in which students find personal meaning in the course. In addition, these data allow us to refine our understanding of those categories. To reflect that refined understanding, we have renamed those categories. For example, in Fig. 4, one of the pathways is labeled "relevant and applicable," rather than "real-world applications." As described in more detail in Sec. IV C ii, this slight change in language reflects the fact that the interviews revealed the importance of personal relevance, not just real-world applicability, in a way that the openended survey questions did not.

In the remainder of this section we explore the pathways by which the students we interviewed described developing a sense of coherence or personal meaning in the course. One can think of each of the arrows leading from low to high engagement in Fig. 4 as a particular engagement pathway, labeled by the factor that is key in facilitating engagement with the curricular material.

Perhaps the most important feature of Fig. 4 is that there are multiple pathways by which students engaged with particular elements of the curriculum. Although it is overly simplistic to associate particular pathways with particular students (individual students surely traverse different pathways at different times and in different settings), it is likely the case that certain student "profiles" are more tightly associated with particular pathways than others. As we will see, some students were not especially interested in coherence across the disciplines, but were interested in materials that had personal meaning. Some students found personal meaning in contexts that had direct relevance to their lives, while others found meaning in contexts that made the world seem more understandable and accessible. Figure 4 is meant to represent these differences, and the multiple ways in which students engage.

The interview data reveal multiple curricular dimensions that shape how students engage with the course. Indeed, the interview data bolster our view, described in Secs. II B and II C, that particular pedagogical features of the course and particular ways in which students were asked to participate in the course were essential for achieving the overall levels of engagement that we observed. In what follows, we narrowly focus our interview analysis on student descriptions of the role that curricular contexts played in fostering interest and engagement.

\section{Coherence as a source of engagement}

Perhaps the most salient feature of the interview data is the excitement that students expressed when describing how the IPLS curriculum fostered a sense of coherence (left-hand side of Fig. 4). Our students frequently expressed deep satisfaction when curricular material supported alternative but complementary understanding of phenomena encountered in biology and chemistry coursework. Bryn, ${ }^{8}$ a student who described the IPLS course as having a particularly profound impact on her understanding of the role of physics in the scientific landscape, described the importance of seeing complementary perspectives on familiar biological problems:

It was definitely familiar material... And I really like learning about the same thing from different perspectives... integrating different perspectives to make more holistic pictures... it's almost as if I previously saw just one half of the picture, and now there's another half... I find it most satisfying when I can look at one thing that I previously saw through one angle, and now look at it through a slightly different angle but still they intersect. They're not just looking at one thing through completely different lenses; they actually converge at some point...

For Bryn, the alternative perspectives encountered in IPLS actually motivated her to seek and use physical explanations in a cell biology seminar she was taking during the same semester as IPLS:

[Prior to IPLS] I didn't really make the connection [between physics and biology], but now, taking a [cell biology] seminar and taking this [IPLS] course, I found that I didn't just apply physics from this course but also just physics in general, what I had previously learned, and just brought it to [cell biology seminar] class, brought it to my [cell biology seminar] professor and said 'this is something interesting I learned today'...

\footnotetext{
${ }^{8}$ All student names are pseudonyms.
} 
In these quotes, Bryn articulates a source of engagement for which the label "interdisciplinary connections" is insufficient. The example quotes in Table IV for the "interdisciplinary connections" label do not necessarily point to new ways of answering questions encountered in other coursework; they merely point to a connection or synergy of some sort between the different courses. For some students this basic connection may be sufficient for serving as a source of engagement. We see with Bryn, however, a layering of explanatory power, one where physical ideas encountered in IPLS provide a new structure for understanding biological problems encountered elsewhere. We use the term "explanatory coherence" to describe the merging of these alternative explanations [31]. Interdisciplinary connections need not have this explicative quality, and in fact such connections can sometimes be quite superficial. Some students find explanatory coherence to be a source of deep engagement, while others articulate a sense of coherence without citing the importance of these layered, alternative explanations. The left-hand side of Fig. 4 depicts these two pathways by which our students described developing a sense of coherence in the IPLS environment.

While not every IPLS student described the course as being as impactful on his or her scientific perspective as did Bryn, the presence in IPLS of alternative explanations for meaningful questions that are familiar from other disciplines was a sentiment articulated by most of the students we interviewed. Of the 13 students interviewed at the end of the IPLS semester, 11 explicitly referenced the importance of being able to connect the IPLS curriculum to specific biology or chemistry courses they had or were currently taking. ${ }^{9}$

Some students did not go into as much detail as Bryn about the merging of alternative explanations, but still described the importance of interdisciplinary connections. Connor, for example, said that modeling the cell membrane as a simple electrical circuit provided a connection to the cell biology course he was taking concurrently with IPLS:

We spent a fair amount of time [in IPLS] on circuits and circuit diagrams and components of circuits... [the instructor] immediately connected it to models of cell membranes and we talked about capacitance and different proteins as being modeled as different circuit components and we really went, we went pretty far with that model... we derived at some point the voltage difference across a membrane and I read [the value for that potential] a couple weeks later in my cell biology textbook and it was pretty close, which was really cool.

\footnotetext{
${ }^{9}$ One of the 14 students interviewed for the study was not interviewed at the end of the IPLS semester, for scheduling reasons.
}

Kyle, a student with particular interests in neurobiology, said that this same IPLS unit on modeling the cell membrane "really played well with" the discussion of the Nernst equation in his neurobiology course. In reflecting on a particular lab in the IPLS course that models action potential propagation down a neural axon, Kyle remarked on the types of explanations that both biology and physics were able to provide. He described the presentation in biology as "trying to understand... the evolutionary and functional perspective" of signal propagation while IPLS provided "the mechanical perspective." Kyle used the term "layering" to describe the way in which physical explanations connected with his understanding from biology and chemistry, and described at length the source of his engagement in particular biomedical applications encountered in the course:

I think in some cases [IPLS] helped me to understand the biology more and in other cases just added another layer of understanding that I didn't know before. I had no clue what NMR was, like I'd encountered it hundreds of times in chemistry but I had no clue... what proton NMR actually was, from a physics basis... Every topic had its sort of own layer of understanding that it added to my understanding of biology and chemistry... I think one of the biggest things this course taught me is understanding that every concept that you learn in biology or chemistry or physics has multiple like, layers of or multiple levels of different subjects integrated with it. So like I can't understand the anatomy of an axon or like the biological properties of an axon without understanding the physics... or I can't really decipher or use a proton NMR machine without really understanding the physics behind it because that doesn't really help me understand why this graph is being produced in the way it is. So I think that's one of the biggest things that [IPLS] has taught us.

The comments from Bryn, Connor, and Kyle show that two of the pathways to engagement entails material that properly leverage students' prior academic experiences in biology and chemistry. We find that curricular choices that foster explanatory coherence, those that support an alternative, complementary understanding of questions encountered in biology and chemistry coursework, resonate strongly with students [31]. Physics materials that only connect to the biological world in a superficial way, but do not help to answer authentic driving questions from other undergraduate coursework, are less successful in engaging students for whom the biological questions are fundamental.

\section{Personal meaning as a source of engagement}

Just as the "interdisciplinary connections" from Table IV can be seen as characterizing one of the pathways to a sense 
of coherence that is important for student engagement, the interview data reveal that "real-world applications" can be viewed as characterizing one of the pathways to a sense of personal meaning. Students described achieving this personal meaning in at least two prominent ways: (i) through biological examples that made the physics applicable and relevant for understanding things they cared deeply about in their lives (this aligns closely with the "real-world applications" label in Table IV, but is not identical because in the context of the interviews the connection to students' personal lives was more apparent) and (ii) through examples that were sensible and understandable, whether or not they were directly relevant to their everyday lives. The right-hand side of Fig. 4 depicts these two engagement pathways.

The unit on human vision and vision correction was met with great enthusiasm, for example, because students were excited to make sense of their own glasses or contact lens prescriptions. Those vision-correcting prescriptions had personal relevance and applicability in a way that microscopy did not. For students aspiring to the health professions, the personal connections around diagnosis and treatment seemed to be especially relevant. Connor described his interest in the vision unit in personal terms:

I also wear glasses so it was interesting to understand what my prescription means and... how you might arrive at that prescription if you're doing an eye exam.

Likewise, the discussion of electrocardiography captured Connor's attention because of his work as an emergency medical technician (EMT):

There was one lab we did [on] electrocardiography, where we had what seems like this really dinky apparatus that you plug in by USB to a computer and put little electrodes on your wrist, but we were able to make these beautiful electrocardiograms that were so, that were really clean and reproducible, which was really interesting to see how easy it is to do that... I've seen a lot of them because I'm an EMT so it was interesting to kind of see how that you know connected [to IPLS]. [EMT trainers] don't actually rigorously teach you how to read them but the paramedics do so I've seen a bunch of them, and I've tried to get them to explain them to me...

Other students similarly described engaging with topics that carried personal meaning for them. When commenting on their interest in medically relevant topics like bonebreaking stress forces or NMR imaging, students were as likely to connect those topics to their own personal experience with sports that they played (and injuries obtained during such sports) as they were to connect the topics to their future career interests in the medical field. Some students who did not express particular interest in the connections to their cell biology or biochemistry coursework, for example, often still expressed interest in material because of its connection to significant experiences in their nonacademic lives.

Not all students who found personal meaning in the curriculum described such meaning in terms of relevance and applicability. Some described personal meaning as stemming from the material being more "understandable" or "accessible." Irene, for example, contrasted a hypothetical study of fluids in which one "just looks at tubes of water and thinks about them" to the IPLS experience of studying fluids in the context of blood flow in the body. She found the blood flow discussion more meaningful because "it's not as abstract" and "makes more sense to me." Irene did not describe the blood flow discussion as relevant for understanding something in her life that she cared deeply about, but she saw the discussion as personally meaningful in that it facilitated her mastering a topic that was otherwise daunting and unreachable. Likewise, Amy described her interest in the discussion of how birds and planes fly-a topic which she had never thought about before and did not carry particular relevance for her-by noting that "now every time I fly in a plane that's all I think about as we go up." Amy described personal meaning in being able to use physics to make sense of phenomena in the world (like flying), even when those phenomena did not touch directly on the things she cared most about in life.

Irene and Amy described their ability to understand the curricular material in retrospective ways. Having internalized the physical ideas, they felt confident that they could use them to make sense of the world. For some students, this ability to understand was described in more prospective terms. Kai, for example, was highly engaged by the discussion of modern physics because it posed a challenge that seemed doable. She described her interest in terms of the personal challenge that the material presented:

I think it's just interesting because it presents a challenge to me intellectually and I want to see if I can do that. I think it's fascinating and I want to give it a shot.

For Kai, modern physics presented a meaningful opportunity to "rise to the occasion" and conquer a genuine intellectual puzzle. The unit on modern physics fostered a sense of agency in her that was not apparent at other moments in the course, and this personal agency seemed important for her engagement with the material.

As we noted at the start of this section, the most important feature of Fig. 4 is that it represents multiple pathways by which students engage with particular elements of the curriculum. In this section we have seen that some students described finding personal meaning in contexts that had direct relevance to their nonacademic lives, while others described finding meaning in contexts that made the world seem more understandable and accessible. Figure 4 is meant to represent these differences, and the multiple ways in which students engage. 
Furthermore, the model in Fig. 4 is not meant to be a comprehensive accounting of all the pathways that are important for student engagement, only those that were most prominent in our interviews with IPLS students at Swarthmore.

\section{DISCUSSION}

Having explored the qualitative survey and interview data from the IPLS students, we are now in a position to better interpret the quantitative example interest rankings from Fig. 2. The high scores students assigned to the "membrane potential" and "nerve signaling" topics are consistent with the finding from the open-ended free responses (Fig. 3) that our students were especially engaged by physics content that made interdisciplinary connections with their other life-science coursework. The electric potential across the cell membrane is explored from a functional perspective in the introductory biology sequence at Swarthmore, in the intermediate-level cell biology course, and in the intermediate-level neurobiology course. The lipid bilayer cell membrane is often touched upon in the general chemistry and biochemistry courses as well, so for many students the building of an electrical circuit model of the cell membrane was the third time they were visiting the question of how cell membranes operate.

Similarly, nerve signaling is a central topic in the intermediate neurobiology course, and frequently is discussed in introductory biology as well. Since most of the students in the course had taken or were currently enrolled in at least some of these courses, the importance of explanatory coherence makes it perhaps unsurprising that students rank the "membrane potential" and "nerve signaling" topics so highly. It is worth noting, however, that it was not the expectation of the IPLS instructors at the outset of the course that these would be among the highest ranked topics. Topics like "electrocardiography," "magnetic sensing," and "pacemaker safety" were incorporated into the course precisely because the curriculum designers expected students to view these as especially interesting applications of the core physics. While the students did express interest in those topics for a variety of reasons, they consistently ranked "membrane potential" and "nerve signaling" as even more interesting.

The data in Table III are further evidence that, rather than viewing the treatment in IPLS as repetitive or unnecessary, the students who had been exposed to an alternative perspective reported being more interested in the topic. Students who could coordinate the IPLS treatment of the dielectric constant to the complementary treatment presented in their biochemistry course ranked the topic more highly on the end-of-semester survey than did their classmates who were seeing the idea of a dielectric constant for the first time. The high scores for "membrane potential" and "nerve signaling," and the different scores given for the "dielectric constant," reflect the most striking feature of both the survey and interview data-the degree to which students described being stimulated by connections to their other biology and chemistry coursework. When tasks and problems connected not just in the abstract with material from the biological or chemical realms, but were actually designed to help students look at questions from biology or chemistry in a new way using new physical tools, students saw their academic experiences as more coherent.

Importantly, physics instructors may not be well positioned to determine which topics their life-science students will find most engaging, let alone to guess which examples will be perceived to be authentic. Instead of relying solely on their own instincts when designing IPLS curricula, these data suggest that consultation with biology and chemistry colleagues who teach the same student population can be valuable. Because many life-science students appreciate opportunities to coordinate physics with the rest of their academic experience, a course that leverages those connections can be especially impactful. Finding out what is actually taught in the biology and chemistry classes, and what instructors of those courses would consider to be the most important and most challenging ideas, is a sensible approach to fostering explanatory coherence in IPLS.

Consider also the students' interest rankings for the "optics of vision" and "optics of microscopy" examples in Fig. 2. While both of these topics involve the same physical principles (those of geometrical optics), and the same amount of time was devoted to each topic in the course, students consistently ranked the "optics of vision" topic as more interesting than they did the "optics of microscopy." They did not report being particularly interested in understanding the optical mechanism underlying microscopy, despite the familiarity that students had with microscopy from their biology laboratory courses, and despite the importance of microscopy in many avenues of biological research. Students were, however, especially interested in unpacking the meaning of an eyeglass lens prescription. This result can perhaps be understood in light of the emphasis that many students placed on being able to attach personal meaning to the examples encountered in the course. Students described being especially interested in the vision and vision-correction examples because they could connect the discussion to their own experiences with vision correction, and in some cases to their future interest in writing prescriptions of various types themselves.

The sources of student interest and engagement in IPLS are complex, involving numerous curricular and pedagogical dimensions, and the students experiencing these dimensions are even more complex themselves. Our curricular materials must therefore have the flexibility to accommodate multiple pathways, multiple ways in which curricular material can lead to engagement.

As IPLS developers continue to design new curricular materials, and as we disseminate such materials to a growing number of IPLS curriculum users, it is important to keep this diversity in mind. It might be advantageous, for 
example, for the IPLS cannon of materials to include a range of problems and activities that are specifically identified as being particularly helpful in facilitating different engagement pathways. This sort of labeling would encourage IPLS adopters to keep in mind the diversity of engagement pathways, and to seek to incorporate materials that meet the needs of as wide a student population as possible.

These pathways may be helpful not just in the construction of tasks and problems, but in the dialogue between instructors and students. As instructors interact and converse with students, these engagement pathways might serve as a set of avenues toward increasing student engagement. In such conversations, instructors could work to scaffold these different engagement pathways and explore which pathways gain traction for which students in particular situations.

\section{FUTURE DIRECTIONS}

While this paper has focused on engagement pathways within IPLS, the pathways in Fig. 4 are not inherently limited to the IPLS environment. Almost all physics courses include curricular materials that are novel to some students and familiar to others. How students respond affectively to novel and familiar material is of relevance to any instructor who hopes to make his or her course interesting and engaging to the widest array of students. Understanding the variety of pathways by which different students become engaged can help to ensure that materials are not being designed too narrowly. It is true that introductory physics courses are the courses most likely to be populated by students with a wide range of academic interests, but it is also certainly true that students in intermediate-level optics courses and upperdivision quantum mechanics classes engage with the curricular content in different ways. We hope that future work will explore these engagement pathways in a variety of physics classroom and laboratory settings and across a broader diversity of major populations.

This paper has largely focused on student data obtained at a particular point in time, at the end of a semester of IPLS physics. Going forward, it will also be important to characterize individual student trajectories, and in particular to associate particular pathways of engagement with different "phases" of interest that students might traverse. Such analysis may allow us to distinguish between ways in which some examples "spark" student interest and others "sustain" student interest [17]. This is not to say that individual students in our study did not describe profound development over the course of their experience in introductory physics, particularly as they moved from the traditional to the IPLS semester. Indeed, one of the most striking holistic results from our analysis of the interviews in this study is the degree to which the IPLS semester changed life-science students' engagement with physics. Such a discussion of development is the focus of future work.

In order to tell such a story of development, we need data that will help us to characterize the trajectory of interest development for particular students during the introductory physics year, and during subsequent biology and chemistry courses and research environments. Such longitudinal studies are essential for understanding whether the interest and engagement described at the end of the IPLS semester is sustained. Do students' experiences with the life-science examples in IPLS influence their approach to upperdivision coursework in their home disciplines? Does interdisciplinary thinking show up in meaningful ways after they have left the IPLS classroom? We already see evidence of this in Bryn's statements, for example, when she describes how physical ideas encountered in the IPLS semester influenced her approach to a cell biology seminar she was enrolled in concurrently. We have yet to explore whether this kind of transfer is true more generally, and whether it persists longitudinally beyond the IPLS course.

\section{ACKNOWLEDGMENTS}

We thank Ann Renninger for her insights regarding educational psychology, and Ann Ruether for assistance with data management. Tessa Williams, Max Franklin, and Haley Gerardi provided useful feedback to initial versions of the central arguments in the paper. This work was supported by a grant to Swarthmore College from the Howard Hughes Medical Institute through the Precollege and Undergraduate Science Education Program, and by NSF DUE-1122941.
[1] O. Bluh, Physics for the biologist, Am. J. Phys. 29, 771 (1961).

[2] P. Argos, General physics course for pre-medical students, Am. J. Phys. 41, 1224 (1973).

[3] E. F. Redish and D. Hammer, Reinventing college physics for biologists: Explicating and epistemological curriculum, Am. J. Phys. 77, 629 (2009).
[4] D. C. Meredith and E. F. Redish, Reinventing physics for life-science majors, Phys. Today 66, 38 (2013).

[5] B. O'Shea, L. Terry, and W. Benenson, From $F=m a$ to flying squirrels: Curricular change in an introductory physics course, Cell Biol. Educ. 12, 230 (2013).

[6] E. F. Redish, C. Bauer, K. L. Carleton, T. J. Cooke, M. Cooper, C. H. Crouch, et al., NEXUS/Physics: An 
interdisciplinary repurposing of physics for biologists, Am. J. Phys. 82, 368 (2014).

[7] Committee on Undergraduate Biology Education, National Research Council, BIO 2010: Transforming Undergraduate Education for Future Research Biologists (National Academies Press, Washington, DC, 2003).

[8] American Association for the Advancement of Science, Vision and Change in Undergraduate Biology Education: A Call to Action (American Association for the Advancement of Science Press, Washington, DC, 2011).

[9] Howard Hughes Medical Institute-American Association of Medical Colleges Committee, Scientific Foundations for Future Physicians (American Association of Medical Colleges, Washington, DC, 2009).

[10] K. L. Hall, Examining the effects of students' classroom expectations on undergraduate biology course reform, Ph.D. thesis, University of Maryland, 2013.

[11] J. Watkins and A. Elby, Context dependence of students' views about the role of equations in understanding biology, CBE-Life Sci. Educ. 12, 274 (2013).

[12] B. D. Geller, J. S. Gouvea, V. Sawtelle, and C. Turpen, Proceedings of the International Conference of the Learning Sciences (International Society of the Learning Sciences, Boulder, CO, 2014), Vol. 1, pp. 1142-1146.

[13] V. Sawtelle and C. Turpen, Leveraging a relationship with biology to expand a relationship with physics, Phys. Rev. ST Phys. Educ. Res. 12, 010136 (2016).

[14] K. A. Renninger and S. E. Hidi, The Power of Interest for Motivation and Engagement (Routledge, New York, 2016).

[15] B. A. Danielak, A. Gupta, and A. Elby, Marginalized identities of sense-makers: Reframing engineering student retention, J. Eng. Educ. 103, 8 (2014).

[16] C. S. Hulleman and J. M. Harackiewicz, Promoting interest and performance in high school science classes, Science 326, 1410 (2009).

[17] S. Hidi and K. A. Renninger, The four-phase model of interest development, Educ. Psychol. 41, 111 (2006).

[18] K. A. Renninger and S. Hidi, Revisiting the conceptualization, measurement, and generation of interest, Educ. Psychol. 46, 168 (2011).

[19] W. K. Adams, K. K. Perkins, N. S. Podolefsky, M. Dubson, N. D. Finkelstein, and C. E. Wieman, New instrument for measuring student beliefs about physics and learning physics: The Colorado Learning Attitudes about Science Survey, Phys. Rev. ST Phys. Educ. Res. 2, 010101 (2006).
[20] C. H. Crouch, P. Wisttanawat, and K. A. Renninger, in Proceedings of the Physics Education Research Conference (American Association of Physics Teachers, Portland, OR, 2013).

[21] C. H. Crouch, P. Wisttanawat, M. Cai, and K. A. Renninger, Life science students' attitudes, interest, and performance in introductory physics for life sciences (IPLS): An exploratory study, Phys. Rev. ST Phys. Educ. Res. 14, 010111 (2018).

[22] K. A. Douglas, M. S. Yale, D. E. Bennett, M. P. Haugen, and L. A. Bryan, Evaluation of Colorado Learning Attitudes about Science Survey, Phys. Rev. ST Phys. Educ. Res. 10, 020128 (2014).

[23] A. Collins, J.S. Brown, and A. Holum, Cognitive apprenticeship: Making thinking visible, Am. Educat. 4, 6 (1991).

[24] C. H. Crouch and K. Heller, Introductory physics in biological context: An approach to improve introductory physics for life science students, Am. J. Phys. 82, 378 (2014).

[25] C. H. Crouch, J. Watkins, A. P. Fagen, and E. Mazur, in Research-Based Reforms in University Physics, edited by E. F. Redish (American Association of Physics Teachers, College Park, MD, 2007), pp. 1-55.

[26] J. E. Watkins, K. L. Hall, J. E. Coffey, T. J. Cooke, and E. F. Redish Disciplinary authenticity: Enriching the reform of introductory physics courses for life science students, Phys. Rev. ST Phys. Educ. Res. 8, 010112 (2012).

[27] R. A. Engle, Framing interactions to foster generative learning: A situative explanation of transfer in a community of learners classroom, J. Learn. Sci. 15, 451 (2006).

[28] R. A. Engle, P. D. Nguyen, and A. Mendelson, The influence of framing on transfer: Initial evidence from a tutoring experiment, Instr. Sci. 39, 603 (2011).

[29] V. Sawtelle and C. Turpen, Leveraging a relationship with biology to expand a relationship with physics, Phys. Rev. ST Phys. Educ. Res. 12, 010136 (2016).

[30] See Supplemental Material at http://link.aps.org/ supplemental/10.1103/PhysRevPhysEducRes.14.010118 for complete list of raw student responses.

[31] B. D. Geller, B. W. Dreyfus, J. S. Gouvea, V. Sawtelle, C. Turpen, and E. F. Redish, Bridging the gaps: Classifying forms of disciplinary boundary crossing in an introductory physics course (to be published). 